OPEN ACCESS

Edited by: Rosario Rizzuto,

University of Padova, Italy

Reviewed by: Kenneth K. W. To,

The Chinese University of Hong Kong, Hong Kong Keith R. Laderoute,

SRI International, USA

*Correspondence:

Margaret Ashcroft

m.ashcroft@medschl.cam.ac.uk

Specialty section: This article was submitted to Molecular and Cellular Oncology, a section of the journal Frontiers in Oncology

Received: 03 February 2017 Accepted: 28 March 2017

Published: 27 April 2017

Citation:

Thomas LW, Staples O, Turmaine M and Ashcroft M (2017) CHCHD4

Regulates Intracellular Oxygenation and Perinuclear Distribution of Mitochondria.

Front. Oncol. 7:71. doi: 10.3389/fonc.2017.00071

\section{CHCHD4 Regulates Intracellular Oxygenation and Perinuclear Distribution of Mitochondria}

\author{
Luke W. Thomas ${ }^{1}$, Oliver Staples ${ }^{2}$, Mark Turmaine ${ }^{3}$ and Margaret Ashcroft ${ }^{1 *}$
}

'Department of Medicine, University of Cambridge, Cambridge Biomedical Campus, Cambridge, UK, ${ }^{2}$ Centre for Cell Signalling and Molecular Genetics, Division of Medicine, University College London, London, UK, ${ }^{3}$ Department of Cell and Developmental Biology, University College London, London, UK

Hypoxia is a characteristic of the tumor microenvironment and is known to contribute to tumor progression and treatment resistance. Hypoxia-inducible factor (HIF) dimeric transcription factors control the cellular response to reduced oxygenation by regulating the expression of genes involved in metabolic adaptation, cell motility, and survival. Alterations in mitochondrial metabolism are not only a downstream consequence of HIFsignaling but mitochondria reciprocally regulate HIF signaling through multiple means, including oxygen consumption, metabolic intermediates, and reactive oxygen species generation. $\mathrm{CHCHD} 4$ is a redox-sensitive mitochondrial protein, which we previously identified and showed to be a novel regulator of HIF and hypoxia responses in tumors. Elevated expression of $\mathrm{CHCHD} 4$ in human tumors correlates with the hypoxia gene signature, disease progression, and poor patient survival. Here, we show that either long-term $(72 \mathrm{~h})$ exposure to hypoxia $\left(1 \% \mathrm{O}_{2}\right)$ or elevated expression of $\mathrm{CHCHD} 4$ in tumor cells in normoxia leads to perinuclear accumulation of mitochondria, which is dependent on the expression of HIF- $1 \alpha$. Furthermore, we show that $\mathrm{CHCHD} 4$ is required for perinuclear localization of mitochondria and HIF activation in response to long-term hypoxia. Mutation of the functionally important highly conserved cysteines within the Cys-Pro-Cys motif of CHCHD4 or inhibition of complex IV activity (by sodium azide) redistributes mitochondria from the perinuclear region toward the periphery of the cell and blocks HIF activation. Finally, we show that CHCHD4-mediated perinuclear localization of mitochondria is associated with increased intracellular hypoxia within the perinuclear region and constitutive basal HIF activation in normoxia. Our study demonstrates that the intracellular distribution of the mitochondrial network is an important feature of the cellular response to hypoxia, contributing to hypoxic signaling via HIF activation and regulated by way of the cross talk between $\mathrm{CHCHD} 4$ and $\mathrm{HIF}-1 \alpha$.

Keywords: hypoxia, hypoxia-inducible factor- $1 \alpha$, mitochondria, mitochondrial localization, perinuclear, coiled-coil helix coiled-coil helix domain containing 4

\section{INTRODUCTION}

Low oxygenation (hypoxia) is a key feature of most human tumors and is associated with tumor progression and treatment resistance. Hypoxia activates a transcriptional program via the hypoxiainducible factor (HIF) dimeric transcription factors, enabling tumor cells to metabolically adapt, survive, and metastasize. As the major sites of oxygen consumption within the cell, mitochondria 
control basal intracellular oxygenation (1) and cell metabolism (2). Mitochondrial reactive oxygen species (ROS) production and respiratory chain function regulate HIF activation (3). In addition, hypoxia and HIF transcriptional targets influence mitochondrial function (4-6). Of particular interest is the recent evidence showing that hypoxia leads to changes in mitochondrial morphology (7) and intracellular distribution (8) that are linked to reduced sensitivity to killing by cytotoxic agents (7) and HIF activation (8), respectively. However, the precise mitochondrial molecular mechanisms involved in tumor cell hypoxia responses that contribute to tumor progression and treatment resistance are not known.

Previously, we discovered that the redox-sensitive mitochondrial protein coiled-coil helix coiled-coil helix ( $\mathrm{CHCH})$ domain containing 4 (CHCHD4) controls basal cellular oxygen consumption rate (OCR), metabolic adaptive responses, HIF activation, and hypoxia signaling in tumor cells (9). We found that CHCHD4 overexpression in human cancers correlates with the hypoxia gene signature and is associated with tumor progression and poor patient survival. CHCHD4 (known as Mia40 in yeast) is a key component of the disulfide relay system (DRS) within the mitochondrial intermembrane space (IMS) (9). As an oxidoreductase and part of the DRS, CHCHD4 functions to import and introduce disulfide bonds into proteins containing highly evolutionarily conserved cysteine motifs [e.g., twin- $\left(\mathrm{CX}_{3} \mathrm{C}\right)$ or twin- $\left(\mathrm{CX}_{9} \mathrm{C}\right)$ motif] that are essential for mitochondrial function (9-12). In doing so, electrons are generated and transferred via the DRS to cytochrome $c$ and complex IV (the molecular oxygen accepting complex) of the respiratory chain (13). CHCHD4 mitochondrial localization and import function are dependent on the highly evolutionarily conserved cysteines within the $\mathrm{CHCH}$ domain and redox-sensitive Cys-Pro-Cys (CPC) motif $(9,10)$. CHCHD4 substrates are involved in various aspects of mitochondrial biology, including the assembly and activity of respiratory chain complexes, modeling of the cristae, lipid biosynthesis, and protein import into the matrix [reviewed in Ref. (12)].

In this study, we evaluate the contribution of $\mathrm{CHCHD} 4$ mitochondrial function to HIF signaling in tumor cells. We show that increased expression of CHCHD4 in tumor cells leads to intracellular hypoxia and constitutive activation of HIF. We show that either long-term exposure of cells to hypoxia (72 h) or increased expression of CHCHD4 in tumor cells leads to (i) the redistribution of the mitochondria to the perinuclear region of the cell, (ii) HIF- $1 \alpha$ stabilization, and (iii) the upregulation of HIF targets. Hypoxic perinuclear localization of mitochondria is dependent on CHCHD4. CHCHD4-mediated perinuclear localization of mitochondria in normoxia correlates with elevated intracellular hypoxia and requires the highly conserved cysteines within the CPC motif. Finally, we show that both hypoxic and CHCHD4-mediated perinuclear accumulation of mitochondria is dependent on HIF- $1 \alpha$ expression. Our data highlight for the first time the importance of $\mathrm{CHCHD} 4$ in regulating mitochondrial subcellular localization, intracellular oxygenation, and HIF activation in tumor cells.

\section{RESULTS}

\section{Hypoxia Induces Perinuclear Localization of Mitochondria}

Mitochondria respond to exogenous hypoxia in numerous ways including regulating oxygen consumption and respiratory chain efficiency (5) and controlling metabolic signaling pathways (14) in order to maintain cellular energy homeostasis and cell viability when oxygen is limiting. In addition, other mitochondrial parameters such as mitochondrial dynamics $(7,15)$ and subcellular localization are also known to be influenced by hypoxia $(8,16)$ via mechanisms that are not yet understood. To explore the cross talk between mitochondria and the hypoxia (HIF) response, we first set out to investigate the effect of hypoxia on mitochondrial morphology and intracellular distribution of the mitochondrial network over a prolonged time course. We found that $72 \mathrm{~h}$ of exposure to $1 \% \mathrm{O}_{2}$, but not 24 or $48 \mathrm{~h}$, led to a visible redistribution of the mitochondrial network from the periphery of the cell to the perinuclear region, in both $\mathrm{HeLa}$ (Figures 1A,B) and in human U2OS osteosarcoma (U2OS-HREluc) cells (Figures 1C,D). U2OS-HRE-luc cells were generated by us previously (17). Digital analysis of the distribution of mitochondrial fluorescence across cytoplasmic segments confirmed a small but significant redistribution of the mitochondria to the perinucleus in HeLa (Figure 1B) and U2OS (Figure 1D) cells. HIF- $1 \alpha$ protein stabilization over this hypoxia time course was strongest at $24 \mathrm{~h}$ in U2OS cells and declined by $48 \mathrm{~h}$, but HIF was still transcriptionally active at $72 \mathrm{~h}$, as indicated by sustained upregulated expression of the HIF targets BNIP3 (Figures 1E,F) and PHD3 (Figure 1F).

\section{CHCHD4 Regulates Perinuclear Localization of Mitochondria and Mitochondrial Morphology}

Recently, we identified the mitochondrial protein CHCHD4 as a critical regulator of HIF- $1 \alpha$ stabilization and signaling in hypoxia through its effects on mitochondrial function (9). For this reason, we next investigated whether CHCHD4 was required for the hypoxic redistribution of mitochondria to the perinucleus. Depletion of CHCHD4 using two independent siRNAs significantly reduced the perinuclear accumulation of the mitochondrial network in cells incubated for $72 \mathrm{~h}$ at $1 \% \mathrm{O}_{2}$ (Figures 2A,B) resulting in a more peripheral distribution of mitochondria (Figure 2C). Consistent with our previous study (9), CHCHD4 knockdown also blocked the hypoxic stabilization of HIF- $1 \alpha$ protein and the transcriptional upregulation of HIF targets, including BNIP3 (Figure 2D) and CA9 (Figure 2E). These data suggest that $\mathrm{CHCHD} 4$ expression is required for the perinuclear redistribution of the mitochondria in response to hypoxia and also indicate that there may be a HIF-dependent aspect to this phenomenon.

Previously, we discovered that $\mathrm{CHCHD} 4$ regulates HIF$1 \alpha$ protein stability and HIF activity in both loss and gain of function systems (9). Therefore, we next investigated whether the mitochondrial network was distributed differently in 

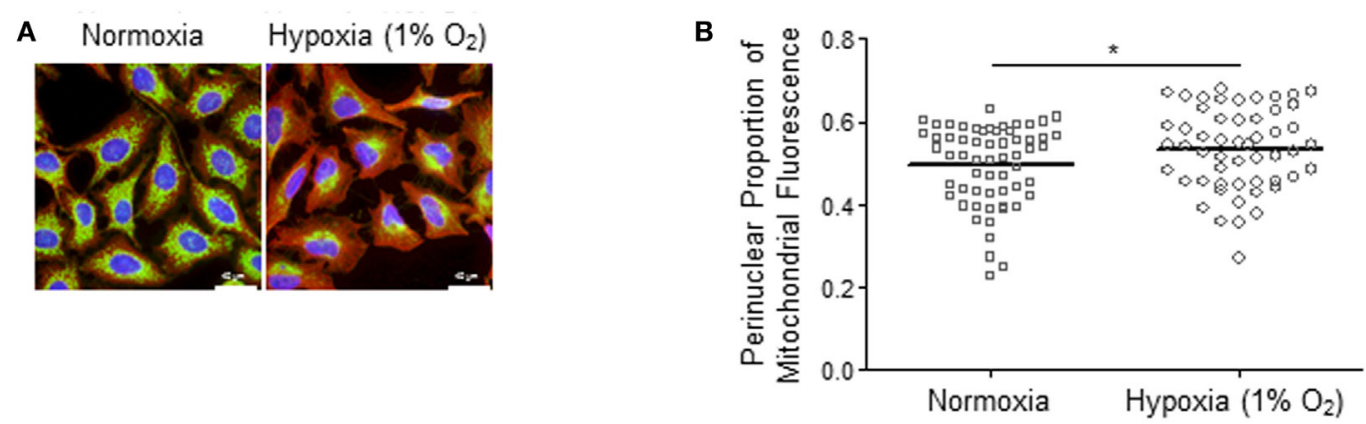

C

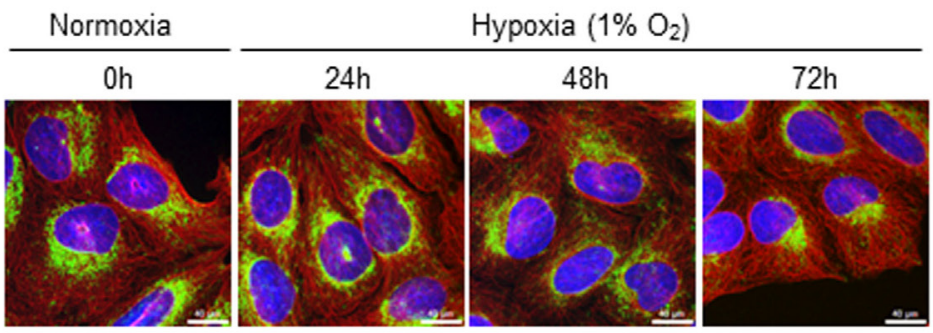

D

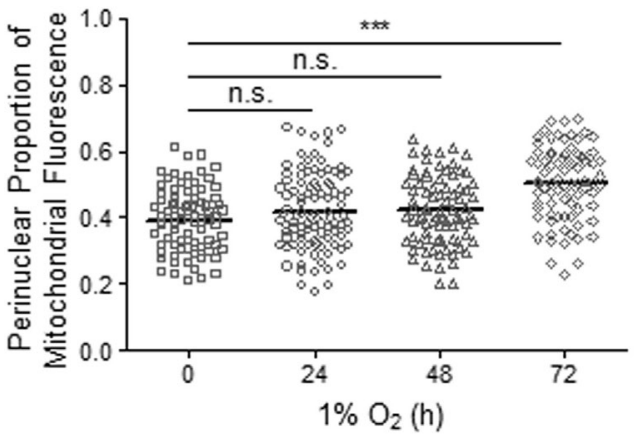

E
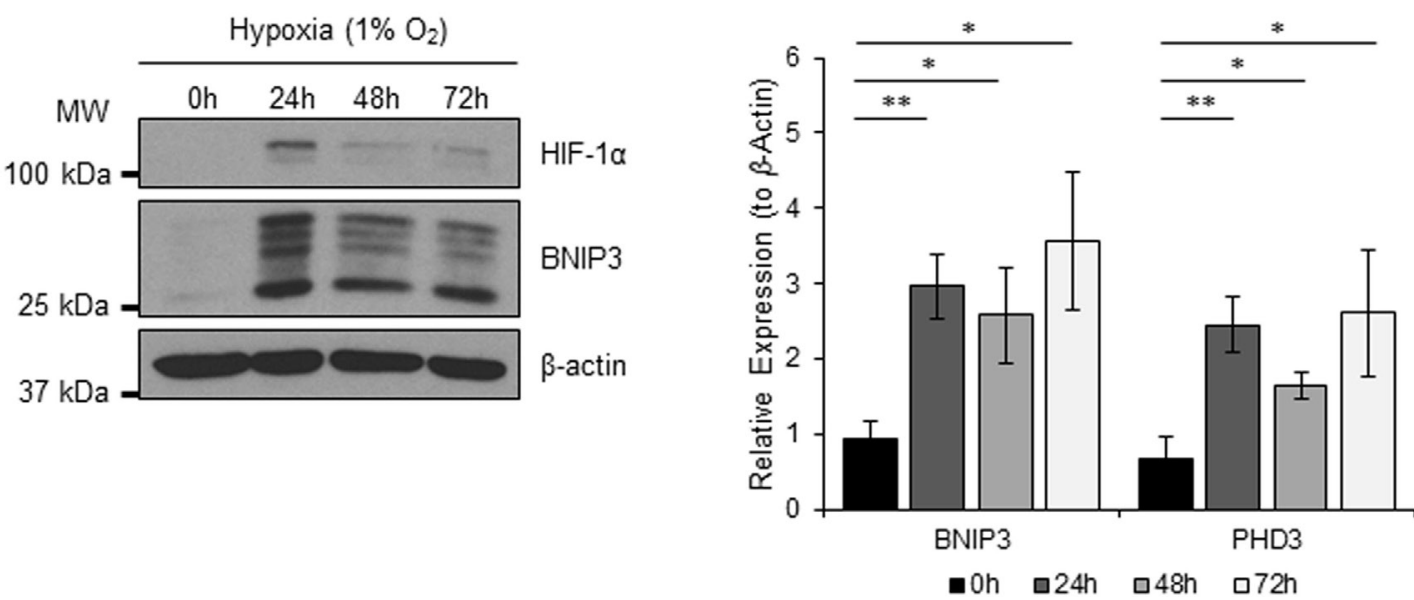

FIGURE 1 | Hypoxia induces perinuclear localization of mitochondria. (A) Images of immunostaining analyses of HeLa cells incubated in normoxia or hypoxia $\left(1 \% \mathrm{O}_{2}\right)$ for $72 \mathrm{~h}$. Cells were immunostained for COXIV (mitochondria, green) and $\alpha$-tubulin (cytoskeleton, red). Nuclei were stained with DAPI (blue). Scale bar $=40 \mu \mathrm{m}$. (B) Graph shows the perinuclear proportion of mitochondrial fluorescence per cell, analyzed from cells described in (A) (10 fields of view per condition). ${ }^{*} p<0.05$. (C) Images of U2OS cells incubated in normoxia or hypoxia $\left(1 \% \mathrm{O}_{2}\right)$ for the indicated times. Cells were immunostained as in (A). Scale bar $=40 \mu \mathrm{m}$. (D) Graph shows the perinuclear proportion of mitochondrial fluorescence per cell, analyzed from cells described in (C) (10 fields of view per condition). $n=3$; n.s., not significant; ${ }^{\star \star *} p<0.001$. (E) Western blots show hypoxia-inducible factor (HIF)- $1 \alpha$ and BNIP3 protein levels in U2OS cells described in (C). $\beta$-actin was used as a load control. (F) Graph shows expression of BNIP3 and PHD3 analyzed by QPCR using total RNA isolated from U2OS cells incubated in normoxia $(0 \mathrm{~h})$ or hypoxia $\left(1 \% \mathrm{O}_{2}\right)$ for 24,48 , and 72 h. ${ }^{*} p<0.05$, ${ }^{\star *} p<0.01$. 

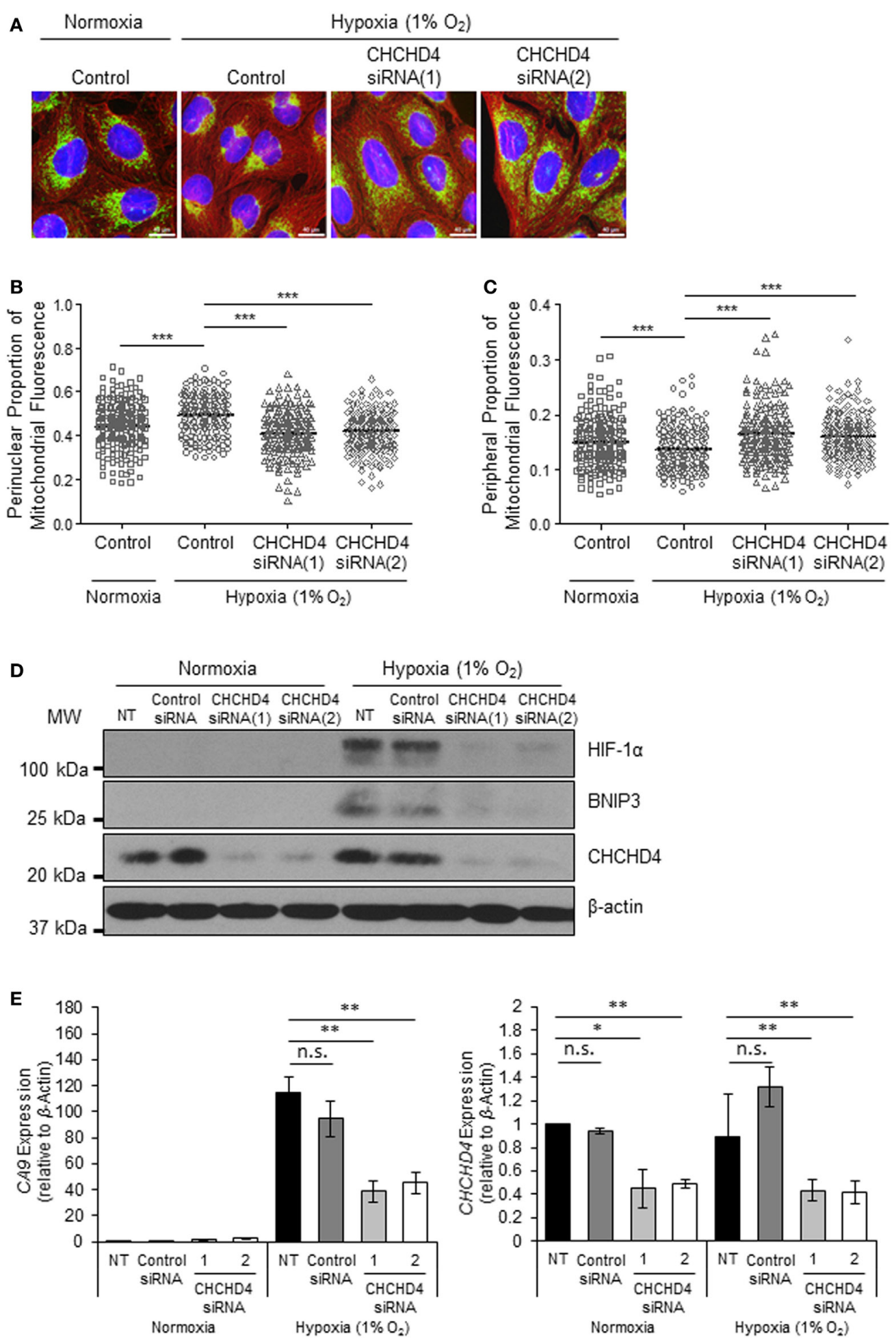

FIGURE 2 | CHCHD4 is required for perinuclear localization of mitochondria in hypoxia. (A) Images of immunostaining analyses of U2OS cells incubated in normoxia or hypoxia $\left(1 \% \mathrm{O}_{2}\right)$ for $72 \mathrm{~h}$. Cells were untreated (control) or transfected with siRNAs targeting CHCHD4 [siRNA(1) and (2)]. Cells were immunostained for COXIV (mitochondria, green) and $\alpha$-tubulin (cytoskeleton, red). Nuclei were stained with DAPI (blue). Scale bar $=40 \mu \mathrm{m}$. (B,C) Graphs show the perinuclear (B) or peripheral $\mathbf{( C )}$ proportion of mitochondrial fluorescence per cell, analyzed from cells described in (A) (10 fields of view per condition). $n=3$; ${ }^{\star \star \star} p<0.001$. (D) Western blots show hypoxia-inducible factor (HIF)- $1 \alpha$, BNIP3, and CHCHD4 protein levels in U2OS cells described in (A). $\beta$-actin was used as a load control. (E) Graphs show expression of $\mathrm{CA} 9$ and $\mathrm{CHCHD} 4$ analyzed by QPCR using total RNA isolated from U2OS cells incubated in normoxia or hypoxia (1\% $\mathrm{O}_{2}$ ) for $72 \mathrm{~h}$. Cells were untreated (NT), treated with non-silencing control (control siRNA) or siRNAs targeting $C H C H D 4[\operatorname{siRNA}(1)$ and $(2)]$. Mean \pm SD. $n=2$. n.s., not significant; ${ }^{* *} p<0.01$. 

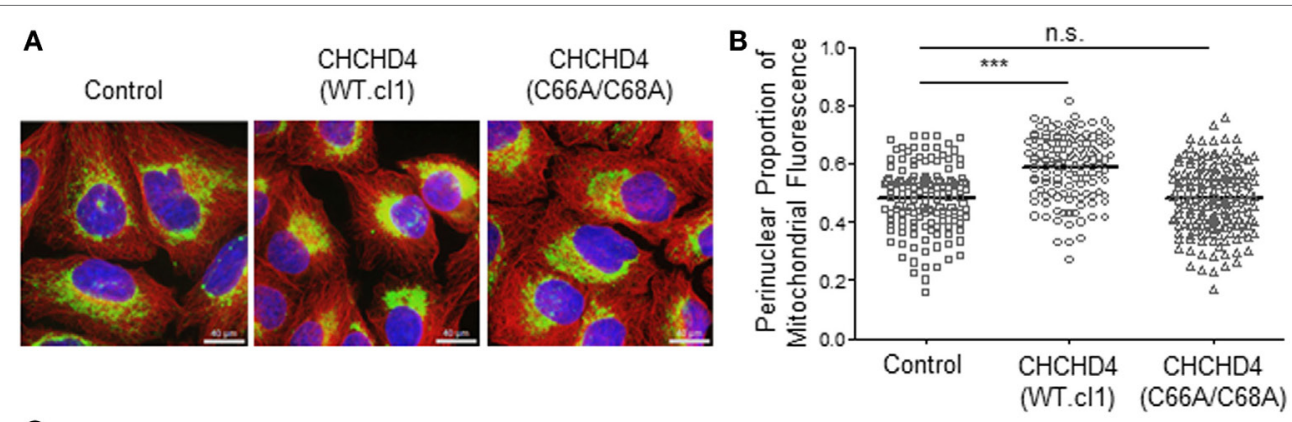

C

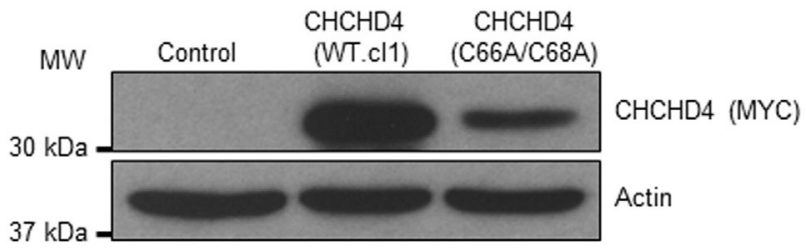

D
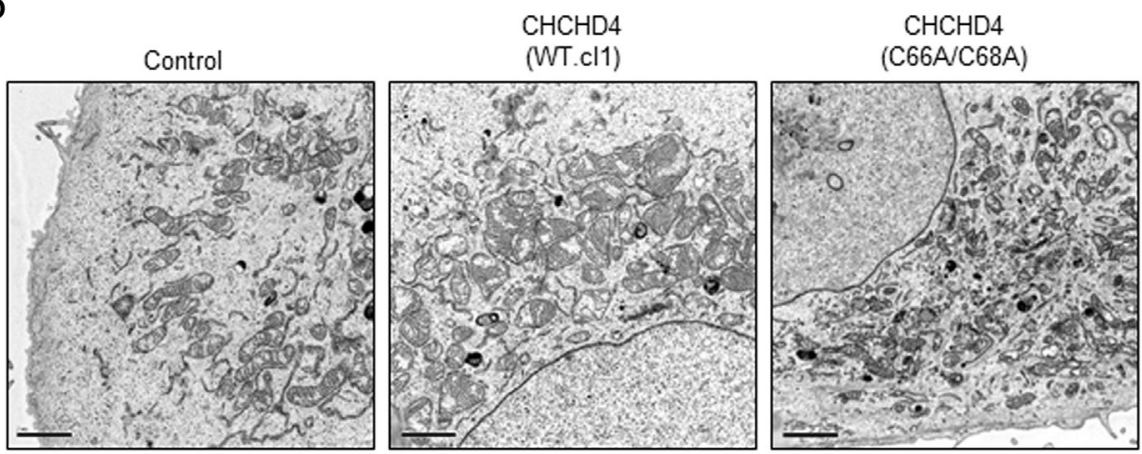

$\mathbf{E}$

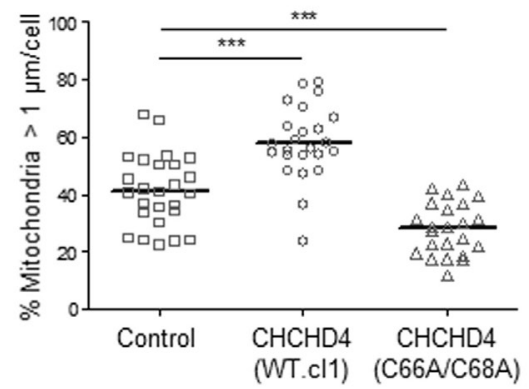

FIGURE 3 | CHCHD4 regulates perinuclear localization of mitochondria and mitochondrial morphology. (A) Images of immunostaining analysis of U2OS cells (control), CHCHD4 wild-type (WT)-expressing (WT.cl1) cells, or CHCHD4 (C66A/C68A)-expressing cells. Cells were immunostained for COXIV (mitochondria, green) and $\alpha$-tubulin (cytoskeleton, red). Nuclei were stained with DAPI (blue). Scale bar $=40 \mu \mathrm{m}$. (B) Graph shows the perinuclear proportion of mitochondrial fluorescence per cell, analyzed from cells described in (A) (10 fields of view per condition). $n=3$; n.s., not significant, ${ }^{\star \star \star} p<0.001$. (C) Western blots show exogenously expressed CHCHD4 protein levels (immunoblotted for the myc-epitope tag) in cells described in (A). $\beta$-actin was used as a load control. (D) Electron microscope images of cells described in (A). Scale bar $=2 \mu \mathrm{m}$. (E) Graph shows \% mitochondria $>1 \mu \mathrm{m} / \mathrm{cell}$ from images described in (D). $n=25$ cells, ${ }^{* * *} p<0.001$.

independent clonal U2OS cell lines stably expressing wild-type (WT) CHCHD4 [CHCHD4 (WT)-expressing cells, clones WT.cl1 or WT.cl3]. Under normoxic conditions, the localization of the mitochondrial network in CHCHD4 (WT)-expressing cells was significantly more perinuclear compared with control cells (Figures 3A-C). However, stable expression of a mutant form of CHCHD4, in which the highly conserved cysteines of the CPC motif have been changed to alanines (C66A/C68A) (9), did not lead to perinuclear accumulation of the mitochondrial network (Figures 3A-C). We (9) and others (10) have previously shown that the highly conserved cysteines within CHCHD4 are required for its mitochondrial localization (9) and import 
function (10). These data suggest that the effects of CHCHD4 on mitochondrial subcellular distribution are dependent on its mitochondrial localization and import function. Interestingly, in both primary human and yeast cells harboring mutations in the sulfhydryl oxidase GFER (Erv1 in yeast), which is essential for the redox activity of CHCHD4 (Mia40 in yeast), the mitochondria show significant changes in both their distribution and morphology $(18,19)$.

Changes in mitochondrial dynamics (fission and fusion) are also known to underlie changes in the distribution of the mitochondrial network and have been detected under both short- (20) andlong-term exposure to hypoxia (7). Using electron microscopy, we confirmed the perinuclear localization of the mitochondria in CHCHD4 (WT)-expressing cells and observed larger mitochondria ( $>1 \mu \mathrm{m}$ in diameter) compared with control cells, suggesting that elevated $\mathrm{CHCHD} 4$ expression causes increased fusion of the mitochondrial network (Figures 3D,E). Consistently, we observed reduced expression of the mitochondrial fission protein dynamin-related protein 1 (DRP-1) (21) in CHCHD4 (WT)expressing cells (data not shown), which is a protein previously shown to be involved in regulating perinuclear localization of mitochondria (15). Conversely, in CHCHD4 (C66A/C68A)expressing cells, we observed a significantly reduced number of larger mitochondria ( $>1 \mu \mathrm{m}$ in diameter) compared to control cells, suggesting a more fragmented phenotype, similar to that described in GFER mutant cells (18).

\section{Elevated CHCHD4 Expression Causes Localized Intracellular Hypoxia within the Perinuclear Region and Constitutive HIF Activation}

It has been shown that the perinuclear accumulation of the mitochondria after short-term exposure to hypoxia is required for the efficient delivery of mitochondrial ROS to the (nuclear) promoters of key HIF target genes such as VEGF. We (9) and others (22) have proposed that mitochondria are responsible for the generation of intracellular oxygen gradients and are associated with local regions of reduced oxygenation within the cell. Furthermore, previously, we have discovered that elevated expression of CHCHD4 in tumor cells leads to increased OCR (9). Therefore, we hypothesized that CHCHD4-mediated perinuclear localization of mitochondria may lead to increased intracellular hypoxia within the perinuclear region of the cell. To investigate this, we used the hypoxia-marker pimonidazole (23) and immunofluorescence microscopy. Notably, pimonidazole adduct formation is oxygen-dependent and occurs independently of changes in pyridine nucleotide redox state (i.e., $\mathrm{NADP} / \mathrm{H}$ ) (24). As anticipated, in CHCHD4 (WT)-expressing cells, we observed increased pimonidazole staining co-localized with mitochondria within the perinuclear region of the cell in normoxia (Figure 4A). We confirmed the elevated perinuclear hypoxia in CHCHD4 (WT)-expressing cells using a second fluorescent hypoxia marker, HypoxiTRAK, by live cell imaging (data not shown). Concurrently, we observed significantly upregulated expression of the HIF-target gene transcripts, CA9 and BNIP3 in the CHCHD4 (WT)-expressing U2OS cells compared to control cells (Figure 4B), indicating above basal HIF-signaling in these cells. Interestingly, we have discovered previously that CHCHD4mediated enhanced HIF- $1 \alpha$ protein stability and HIF activation is sensitive to inhibition by the complex IV inhibitor, sodium azide (9). Therefore, next, we assessed whether CHCHD4-mediated perinuclear localization of mitochondria was also affected by sodium azide treatment. Indeed, we found that sodium azide inhibited complex IV activity (data not shown) and significantly reduced the perinuclear localization of mitochondria (Figure 4C) and associated intracellular hypoxia (data not shown) in the CHCHD4 (WT)-expressing cells. Collectively our data suggest that $\mathrm{CHCHD} 4$-mediated perinuclear accumulation of mitochondria is capable of generating associated localized areas of intracellular hypoxia within the perinuclear region of the cell that are sufficient to lead to HIF transcriptional activation in normoxia. Furthermore, these effects are dependent on respiratory chain function (at least in part via complex IV).

\section{Perinuclear Accumulation of Mitochondria Is HIF-1 $\alpha$-Dependent}

The HIF transcription factor family constitute the major molecular regulators of the cellular response to hypoxia $(25,26)$ and are known to upregulate the expression of proteins involved in mitochondrial morphology and distribution (16). Therefore, next, we investigated whether perinuclear redistribution of mitochondria in response to prolonged hypoxia was dependent on HIF- $1 \alpha$ expression. Interestingly, we found that siRNA-mediated depletion of HIF- $1 \alpha$ blocked the perinuclear accumulation of mitochondria in U2OS cells incubated for $72 \mathrm{~h}$ in $1 \% \mathrm{O}_{2}$ (Figures 5A-C). HIF- $1 \alpha$ knockdown also blocked hypoxic upregulation of the HIF transcriptional target BNIP3 (Figure 5C). Furthermore, depletion of HIF-1 $\alpha$ using two independent siRNAs reversed the perinuclear accumulation of mitochondria in CHCHD4 (WT)-expressing cells (Figures 6A,B). However, we observed no significant effect of HIF- $1 \alpha$ siRNA knockdown on the distribution of mitochondria in control cells (Figures 6A,B), despite significant knockdown of HIF1A expression (Figure 6C).

Our data highlight $\mathrm{CHCHD} 4$ as a critical regulator of mitochondrial distribution and morphology. Perinuclear accumulation of mitochondria in response to hypoxia or elevated CHCHD4 is associated with increased intracellular hypoxia within the perinuclear region and elevated HIF- $1 \alpha$ stabilization and HIF transcriptional activation. Taken together, our study indicates that $\mathrm{CHCHD} 4$ can control the distribution of the mitochondrial network in tumor cells through its role in regulating respiratory chain function, intracellular oxygenation, and HIF activation.

\section{MATERIALS AND METHODS}

\section{Cell Culture}

Human U2OS osteosarcoma (U2OS-HRE-luc) cells have been described by us previously (17) and stably express a luciferase reporter construct under the control of a hypoxia response element, allowing us to monitor HIF/HRE activity alongside 
A

$$
\begin{array}{ll}
\text { Pimonidazole (FITC) } & \text { ATP5B (TRITC) } \\
\text { DAPI (blue) } & \text { DAPI (blue) }
\end{array}
$$
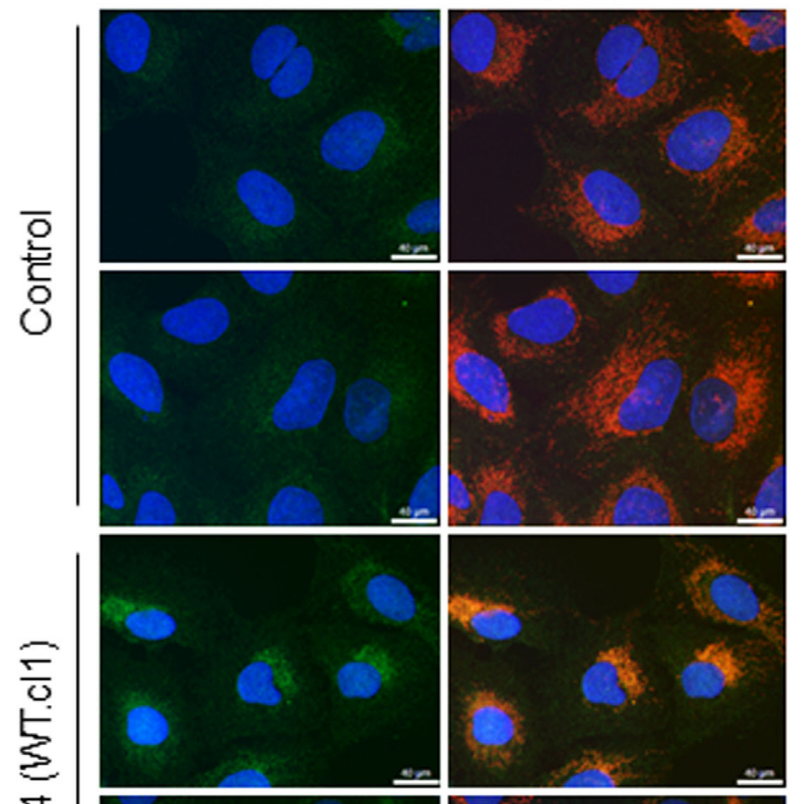

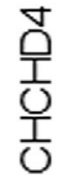
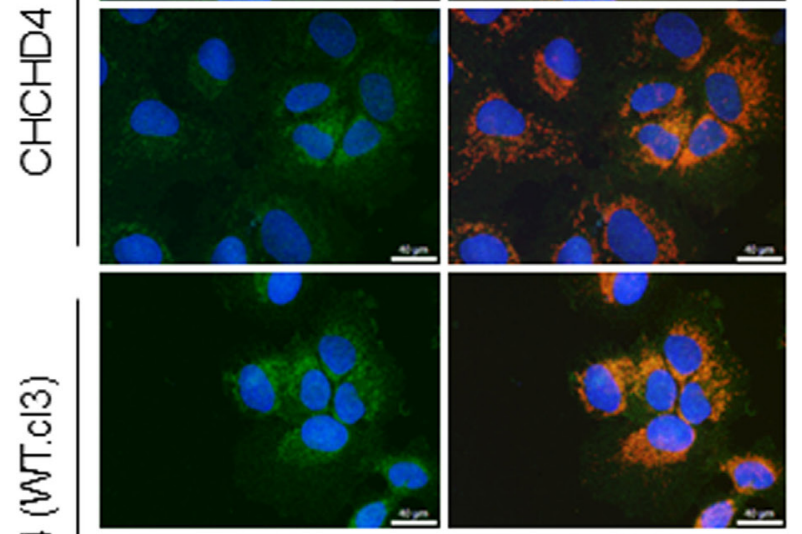

몽
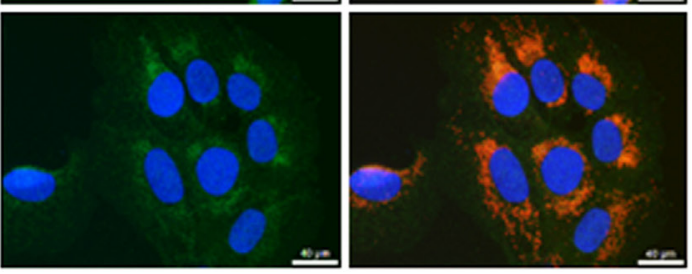

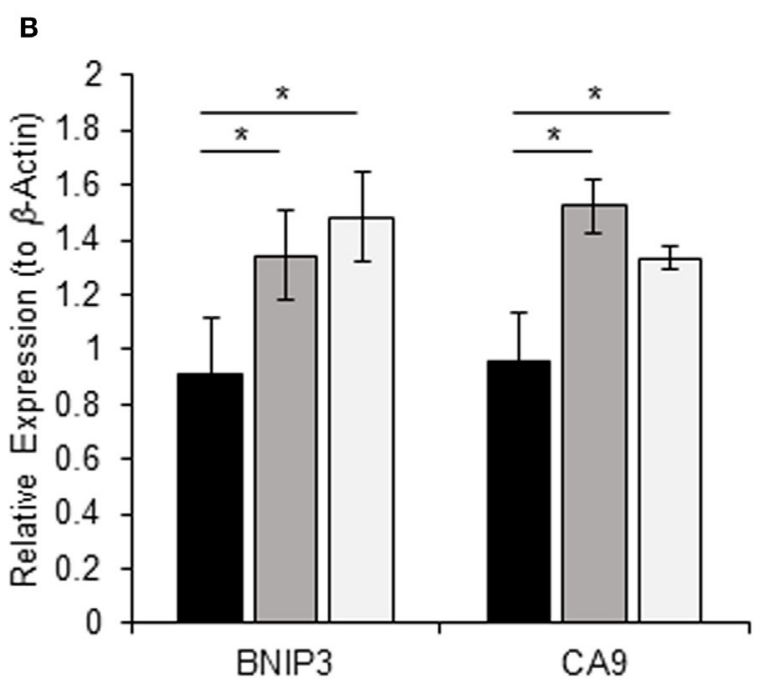

- Control $\square \mathrm{CHCHD} 4$ (WT.cl1) $\mathrm{aCHCHD4}$ (WT.cl3)

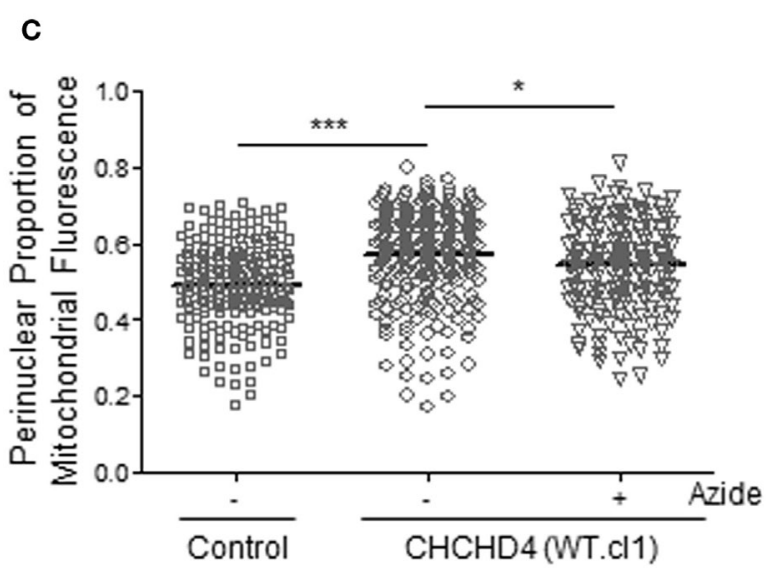

FIGURE 4 | Elevated CHCHD4 expression causes localized intracellular hypoxia within the perinuclear region and constitutive hypoxia-inducible factor activation. (A) Images of immunostaining of U2OS cells (control) and CHCHD4 wild-type (WT)-expressing cells (clones WT.cl1 and WT.cl3) incubated with pimonidazole for $2 \mathrm{~h}$. Cells were immunostained for pimonidazole (green) and ATP5B (mitochondria, red). Nuclei stained with DAPI (blue). Scale bar = $40 \mu \mathrm{m}$. (B) Graph shows expression of BNIP3 and CA9 analyzed by QPCR using total RNA isolated from cells described in (A). Mean \pm SD. ${ }^{*} p<0.05$. (C) Graph shows the perinuclear proportion of mitochondrial fluorescence per cell, analyzed from cells described in (A), after treatment without $(-)$ or with (+) sodium azide (azide, $5 \mathrm{mM}$ ) for $16 \mathrm{~h}$ (10 fields of view per condition). $n=3$; ${ }^{\star \star \star} p<0.001,{ }^{\star} p<0.05$.

measuring mitochondrial endpoints. U2OS osteosarcoma (U2OS) cells were used to generate stable independent clonal cell lines (WT.cl1 and WT.cl3)-expressing CHCHD4.1 cDNA (CHCHD4 (WT)-expressing cells), CHCHD4-C66A/C668A cDNA (CHCHD4 (C66A/C68A)-expressing cells) or pcDNA3.1 (control) by neomycin selection with G418, using constructs we have described previously (9). HeLa cells were purchased from ATCC. All cell lines were maintained in Dulbecco's modified eagle medium containing glucose (4.5 g/L) (Life Technologies) and supplemented with $10 \%$ fetal calf serum (FCS, SeraLabs), penicillin $(100 \mathrm{IU} / \mathrm{mL})$, streptomycin $(100 \mu \mathrm{g} / \mathrm{mL})$, and glutamine $(6 \mathrm{mM})$, all purchased from Life Technologies. Cell lines used were authenticated and routinely confirmed to be negative for any Mycoplasma 

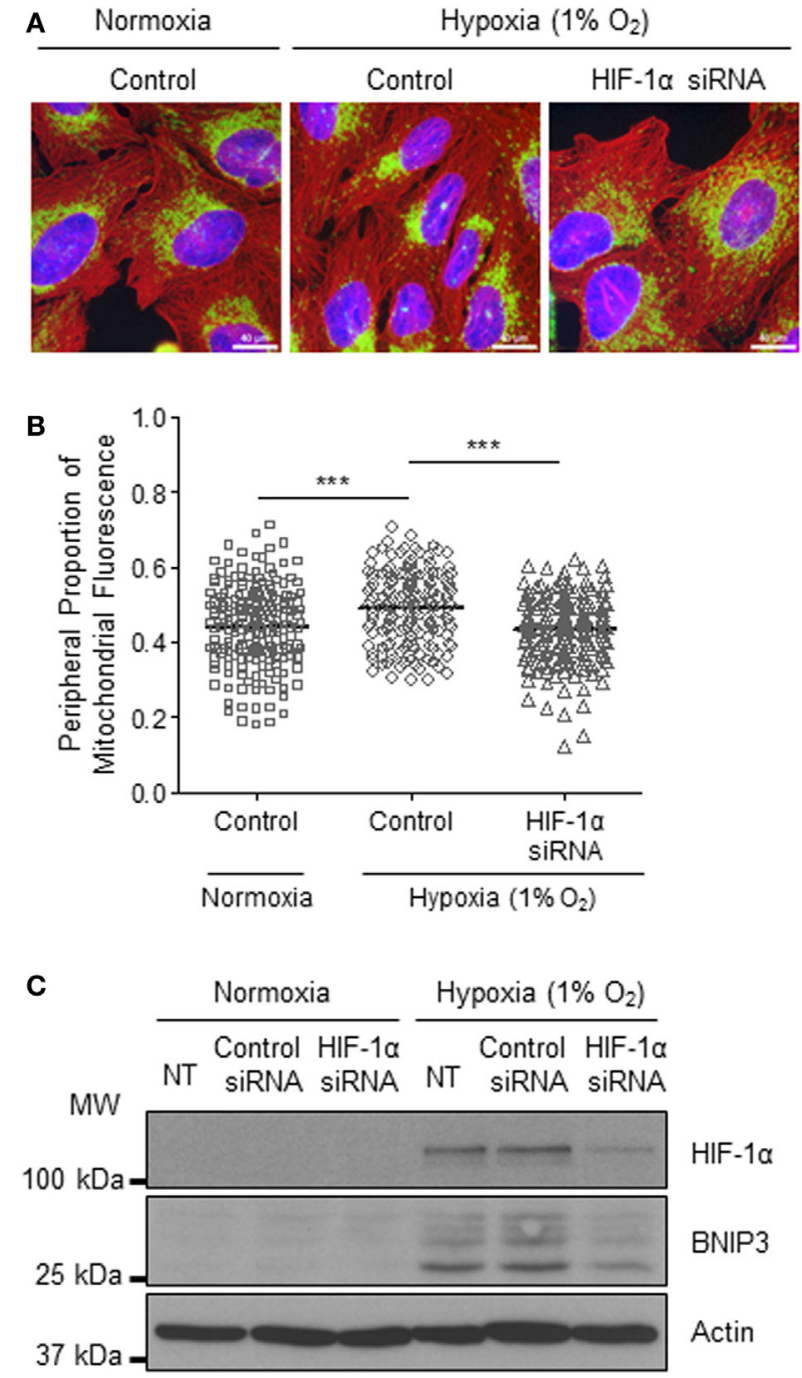

FIGURE 5 | Hypoxia-inducible factor (HIF)-1 $\alpha$ knockdown reverses hypoxia-induced perinuclear localization of mitochondria.

(A) Images of immunostaining of U2OS cells incubated in normoxia or hypoxia $\left(1 \% \mathrm{O}_{2}\right)$ for $72 \mathrm{~h}$. Cells were untreated (control) or transfected with siRNA targeting HIF1A (HIF-1 $\alpha$ siRNA). Cells were immunostained for COXIV (mitochondria, green), and $\alpha$-tubulin (cytoskeleton, red). Nuclei were stained with DAPI (blue). Scale bar $=40 \mu \mathrm{m}$. (B) Graph shows the perinuclear proportion of mitochondrial fluorescence per cell, analyzed from cells described in (A) (10 fields of view per condition). $n=3$; ${ }^{* * *} p<0.001$. (C) Western blots show HIF-1 $\alpha$ and BNIP3 protein levels in U2OS cells untreated (NT), or transfected with a non-silencing control (control siRNA) or siRNA targeting HIF1A (HIF-1 $\alpha$ siRNA). Cells were incubated in normoxia or hypoxia $\left(1 \% \mathrm{O}_{2}\right)$ for $72 \mathrm{~h}$. $\beta$-actin was used as a load control.

contamination. Hypoxia was achieved by incubating cells in $1 \% \mathrm{O}_{2}, 5 \% \mathrm{CO}_{2}$, and $94 \% \mathrm{~N}_{2}$ in a Ruskinn SCI-tive workstation, without agitation.

\section{Antibodies and Reagents}

The rabbit polyclonal $\mathrm{CHCHD} 4$ and rabbit polyclonal BNIP3 antibodies were purchased from Cambridge Biosciences. The mouse monoclonal HIF- $1 \alpha$ antibody was purchased from BD Biosciences. The mouse monoclonal $\beta$-actin and rabbit polyclonal ATP5B antibodies were purchased from Abcam. The mouse monoclonal anti-myc (9B11) and rabbit monoclonal COXIV (3E11) antibodies were purchased from Cell Signaling Technology. The mouse monoclonal anti-pimonidazole antibody was purchased from HypoxyProbe, Inc. The donkey anti-rabbit and anti-mouse horseradish peroxidase-linked secondary antibodies were purchased from VWR. Sodium azide was purchased from SigmaAldrich and used at $5 \mathrm{mM}$ for $16 \mathrm{~h}$ in order to optimally block HIF$1 \alpha$ protein levels without significantly affecting cell viability. DAPI solution $(10 \mathrm{mg} / \mathrm{mL})$ was purchased from Cambridge Biosciences. Pimonidazole (200 mM) was purchased from HypoxyProbe, Inc.

\section{Immunofluorescent Microscopy}

Cells were seeded onto $13 \mathrm{~mm} \varnothing$ coverslips, and after treatment, were fixed in either $4 \%$ paraformaldehyde (PFA) for $15 \mathrm{~min}$ (for assessment of intracellular hypoxia by pimonidazole staining) or $100 \%$ ice-cold methanol overnight (for assessment of the mitochondrial distribution by COXIV or ATP5B). Notably, we found that COXIV and ATP5B total protein levels in cells were not significantly affected by either prolonged exposure to hypoxia $\left(1 \% \mathrm{O}_{2}\right)$ or stable increased expression of $\mathrm{CHCHD} 4$ and as such, we deemed these mitochondrial proteins as suitable markers for immunostaining mitochondria in the experiments described in this study. For PFA fixed samples, cells were washed and permeabilized with a $0.5 \%$ Triton-X solution for $10 \mathrm{~min}$, then washed with phosphate-buffered saline. Immunostaining was carried out using primary antibodies followed by fluorescently labeled secondary antibodies (anti-mouse Alexa 568 and anti-rabbit Alexa 488, Life Technologies) as well as DAPI. All cell imaging was carried out using a DMI4000 B inverted microscope (Leica).

\section{Mitochondrial Distribution Analysis}

Mitochondrial distribution analyses was carried out using Cell Profiler Image analysis software (27), and a custom image analysis pipeline was applied to each data set, as follows: images were loaded, and blue, green, and red channels were split into separate greyscale images and named "nuclei," "mitochondria," and "cell," respectively. Individual, whole DAPI-stained (blue) fluorescent nuclei were identified as objects between 50 and 450 pixel units in diameter. Cell boundaries were identified using anti- $\alpha$-tubulin (red) fluorescence, with Ridler-Calvard thresholding adopted to distinguish between cell edges. Only images of whole, entire nuclei, and/or whole entire cells were included. Nuclei or cells with their boundaries touching the edge of the image were excluded. Nuclear (blue) fluorescence was subtracted from the associated cell fluorescence with the resulting fluorescent shape identified as the cytoplasmic compartment. Each cytoplasmic compartment was divided into five equally spaced concentric rings (to control for different cell size), starting from the boundary of the nucleus. The mitochondrial (green) fluorescence intensity was measured in each concentric ring and provided as a fraction of the total cytoplasmic compartment fluorescence per cell. The sum of the two concentric areas closest to the nuclei was taken as the perinuclear region, while the two outermost concentric areas were taken 
A
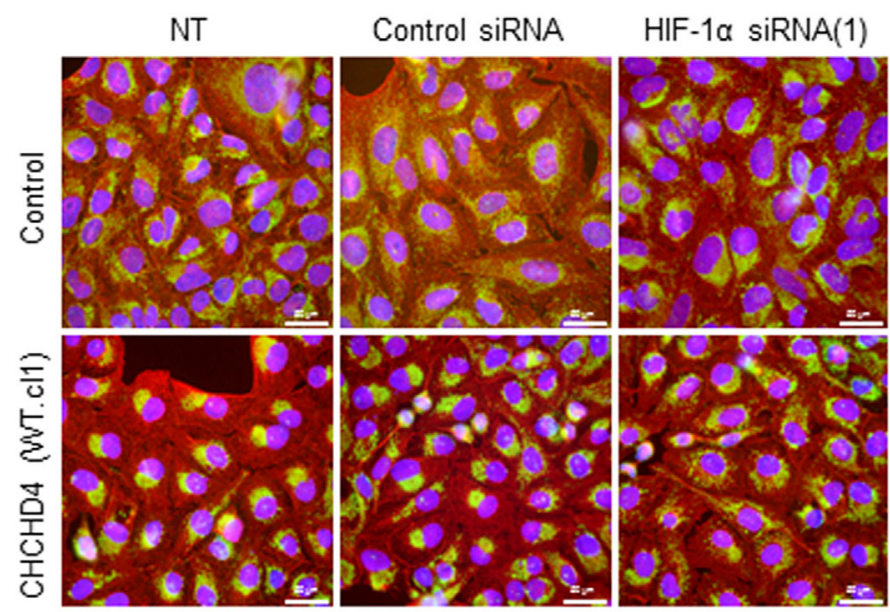

HIF-1 $\alpha$ siRNA(2)
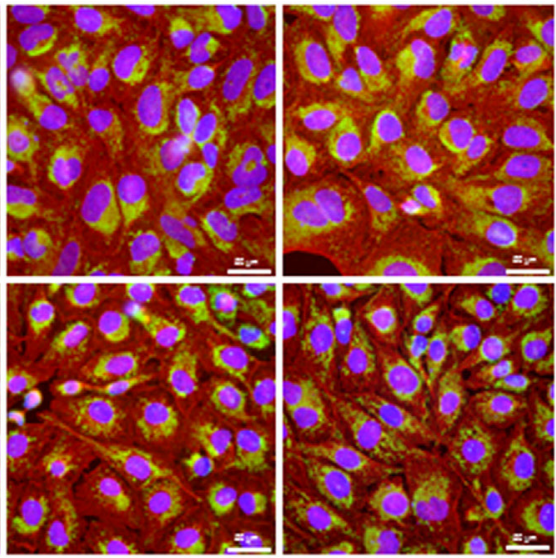

B

Control

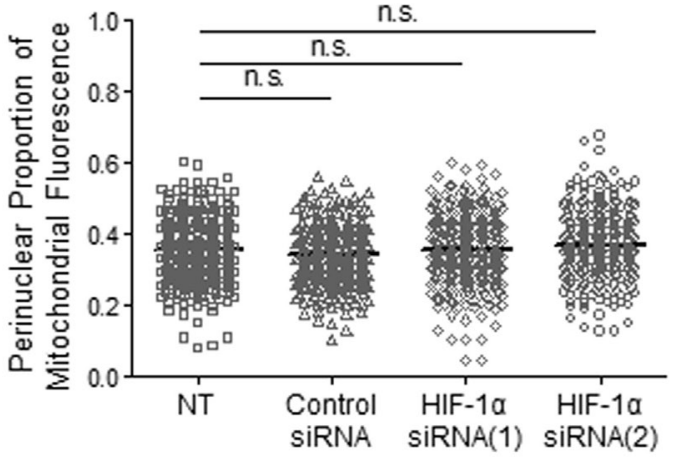

CHCHD4 (WT.cl1)

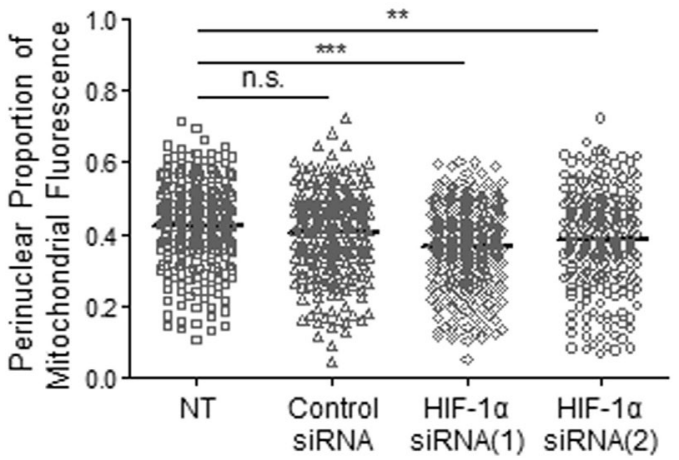

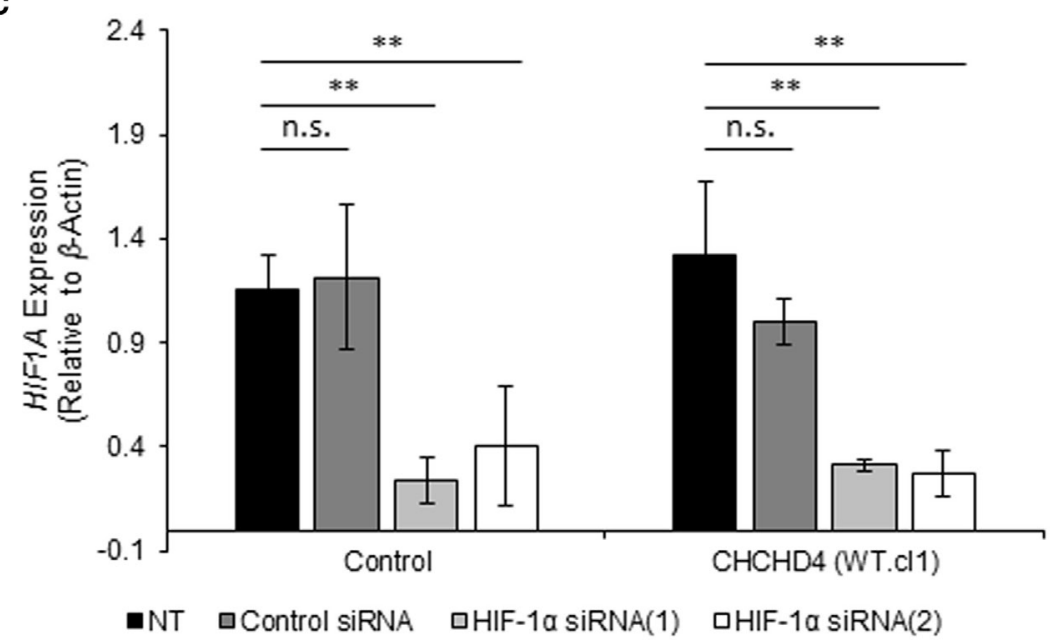

FIGURE 6 | Hypoxia-inducible factor (HIF)-1 $\alpha$ knockdown reverses CHCHD4-induced perinuclear localization of mitochondria. (A) Images show contro U2OS cells (control) and CHCHD4 (WT)-expressing cells (WT.cl1), which were untreated (NT) or transfected with siRNAs targeting HIF1A [HIF-1 $\alpha$ siRNA (1) and (2)]. Cells were immunostained for COXIV (mitochondria, green) and $\alpha$-tubulin (cytoskeleton, red). Nuclei were stained with DAPI (blue). Scale bar $=50 \mu m$. (B) Graphs show the perinuclear proportion of mitochondrial fluorescence per cell, analyzed from cells described in (A) [10 fields of view for each control cells (left graph), and CHCHD4 WT-expressing cells (WT.cl1, right graph)]. $n=3$; n.s., not significant; ${ }^{\star \star \star} p<0.001$. (C) Graph shows expression of HIF1A analyzed by QPCR using total RNA isolated from cells described in (A). Mean \pm SD. $n=3$. n.s., not significant; ${ }^{* \star} p<0.01$. 
as the peripheral region. At least 10 fields of view were analyzed per condition, per experiment, and all data points expressed with mean indicated (solid black bar).

\section{Electron Microscopy}

Cells were seeded onto glass coverslips ( $\varnothing 13 \mathrm{~mm}$, VWR) and fixed in $2 \%$ PFA and $1.5 \%$ glutaraldehyde in $0.1 \mathrm{M}$ cacodylate buffer. Samples were post-fixed in $1 \% \mathrm{OsO}_{4}$ (osmium tetraoxide) and $1.5 \%$ potassium ferrocyanide $\left[\mathrm{K}_{4} \mathrm{Fe}(\mathrm{CN})_{6}\right]$ in $0.1 \mathrm{M}$ cacodylate buffer. Samples were dehydrated using a graded ethanol-water series, cleared in propylene oxide, and infiltrated with agar-100 resin. Ultrathin sections were cut and collected on 300-mesh grids and stained with lead citrate. Sections were viewed using a Joel 1010 transition electron microscope and images were recorded using a Gatan Orius CDD camera. Mitochondrial section diameter was determined relative to the scale bar, and mitochondria grouped above and below $1 \mu \mathrm{m}$ diameter. At least 20 fields of view were analyzed per condition.

\section{Gene Silencing}

Non-silencing siRNA duplexes (universal negative control \#1) and custom designed siRNA duplexes were purchased from SigmaAldrich and transfected into sub-confluent cells using HiPerfect transfection reagent (QIAGEN) according to the manufacturer's instructions. The target sequences for $\mathrm{CHCHD} 4$ were $5^{\prime}$-GAGG AAACGTTGTGAATTA-3' (siRNA(1)) and 5'-AAGATTTGGAC CCTTCCATTC-3' (siRNA(2)). The target sequences for HIF1A were $5^{\prime}$-TACGTTGTGAGTGGTATTATT-3' (siRNA(1)) and 5'-TAGA AGGTATGTGGCATTTAT-3' (siRNA(2)).

\section{Gene Expression Analysis}

Total RNA samples were isolated using the GeneElute kit and protocol (Sigma-Aldrich). cDNA synthesis was carried out using the qScript synthesis kit and protocol (Quantabio). mRNA expression was measured by quantitative (Q)PCR using SYBR Green Mastermix (Eurogentec Ltd.) and the DNA Engine Opticon 2 system (BioRad). The QPCR primer sequences were as follows: CHCHD4_F 5'-GAGCTGAGGAAGGGAAGGAT-3', CHCHD4_R 5'-AATCCATGCTCCTCGTATGG-3'; HIF1A_F 5'-TCCAAGAAGCCCTAACGTGT-3', HIF1A_R 5'-TGATCGTCT GGCTGCTGTAA-3'; CA9_F5'-GCCGCCTTTCTGGAGGA-3', CA9_R 5'-TCTTCCAAGCGAGACAGCAA-3'; BNIP3_F 5'-GAT ATGGGATTGGTCAAGTCG-3', BNIP3_R 5'-CGCTCGTG TTCCTCATGCT; ACTB_F 5'-CCCAGAGCAAGAGAGG-R', $A C T B \_$R 5'-GTCCAGACGCAGGATG.

\section{Statistics}

Values are expressed as mean \pm SD unless otherwise stated and statistical significance was determined by a 2 -tailed unpaired Student's $t$-test, with significance set at $p<0.05$.

\section{DISCUSSION}

Mitochondria are organelles that exist in dynamic interrelated forms within a cellular mitochondrial network (28). It is now becoming clear that as such, mitochondria can influence many cellular processes and communicate with other organelles and intracellular machinery through changes in mitochondrial dynamics (fission and fusion), subcellular localization and morphology (29). As the major sites of oxygen consumption within the cell, precisely how changes in intracellular oxygenation affect mitochondrial behavior is of particular interest since hypoxia underlies the pathology of a broad range of diseases including cancer.

Previous studies in various cell contexts have shown that hypoxia induces a redistribution of mitochondria to the perinuclear region of the cell $(8,15,20,30)$ and regulates mitochondrial dynamics and morphology, promoting enlarged, donut-like (also known as toroid) mitochondria $(7,20,31)$. Here, we have demonstrated that hypoxia stimulates perinuclear accumulation of mitochondria in a $\mathrm{CHCHD} 4$-dependent manner.

We identified human $\mathrm{CHCHD} 4$ previously using a functional genomics approach and showed that it is crucial for regulating hypoxic (HIF) responses, basal OCR and metabolism in tumor cells (9). CHCHD4 is involved in importing and folding $\mathrm{CHCH}$-domain-containing mitochondrial proteins as part of the DRS within the IMS (12) and in doing so, links the DRS to respiratory chain function. Interestingly, mutations in the sulfhydryl oxidase GFER (which recycles CHCHD4 within the DRS) are associated with changes in mitochondrial morphology via changes in the mitochondrial recruitment of the fission protein DRP-1 (18).

We discovered previously that increased expression of CHCHD4 in human cancers (e.g., breast, glioma, and pancreatic) is associated with the upregulation of hypoxia regulated genes and poor patient survival (9). Here, we found that increased expression of CHCHD4 in tumor cells resulted in perinuclear accumulation of the mitochondria, intracellular hypoxia associated with the perinuclear region and constitutive HIF activation in normoxia. Hypoxia within the tumor microenvironment is a well-known driver of tumor cell invasiveness and resistance to therapy (32), mediated primarily through the upregulation of HIF targets that enable tumor cells to survive and metabolically adapt $(25,26)$. Mitochondria are uniquely placed to influence intracellular oxygenation and communicate to HIF. Indeed, the ability for mitochondria to localize within close proximity to the nucleus in response to hypoxia has been proposed to enable mitochondrial ROS to accumulate within the nucleus and enhance the transcription of genes such as VEGF through promoter oxidation (8). Our study describes for the first time that regional (perinuclear) intracellular hypoxia is associated with increased basal transcription of HIF target genes.

Interestingly, we found that perinuclear mitochondria in CHCHD4 (WT)-expressing cells could be redistributed toward the periphery of the cell by transient (siRNA) depletion of HIF- $1 \alpha$ or by treatment with the complex IV inhibitor, sodium azide. Notably, we found that the intracellular hypoxia observed in $\mathrm{CHCHD} 4$ (WT)-expressing cells was reduced by sodium azide treatment, while depletion of HIF- $1 \alpha$ had no significant effect (data not shown). Based on our findings we propose that CHCHD4's upstream control of respiratory chain function and basal OCR (at complex IV) is important for regulating intracellular oxygenation and HIF- $1 \alpha$ signaling, while perinuclear localization of mitochondria is downstream. 
As well as HIF- $1 \alpha$, HIF- $2 \alpha$ protein is also dynamically induced over time in hypoxia, and it will be important to establish what role, if any, HIF- $2 \alpha$ might play in regulating mitochondrial distribution and morphology. Furthermore, the response to hypoxia includes HIF-dependent metabolic adaptations which involve substrate switching and the reprogramming of metabolic pathways to maintain energetic and biosynthetic homeostasis (33). Thus, the prolonged time-frame for perinuclear accumulation of the mitochondria to occur in hypoxia $(72 \mathrm{~h})$ may reflect the metabolic and bioenergetic consequences of HIF activation. For example, in hypoxia, glucose consumption is elevated to support anaerobic ATP generation, while glutamine is diverted from the TCA cycle, and undergoes reductive carboxylation to support the production of lipids for membrane biosynthesis, both of which depend on HIF-1 signaling. Extended culture of cells in hypoxia is likely therefore to lead to the depletion of different metabolic substrates from, and accumulation of different metabolic by-products (e.g., lactate) in the culture medium compared to cells cultured in normoxia. It has been shown that substrate starvation has significant effects on mitochondrial motility (34) and morphology (35), and these two parameters are known to be intimately linked to the bioenergetic status of the mitochondria. Therefore, it will be of interest to investigate whether metabolic substrate availability (increased or decreased) is able to affect perinuclear accumulation of the mitochondria in response to changes in intracellular oxygenation.

Understanding the molecular mechanisms underlying how tumors respond to hypoxia is of great importance both in devising prognostic tests and for identifying improved therapeutic strategies for cancer treatment. Indeed, recent studies have highlighted advances in imaging mitochondrial dynamics associated with hypoxia in tumors for potential use in diagnosis (36) or as a

\section{REFERENCES}

1. Kurokawa H, Ito H, Inoue M, Tabata K, Sato Y, Yamagata K, et al. High resolution imaging of intracellular oxygen concentration by phosphorescence lifetime. Sci Rep (2015) 5:10657. doi:10.1038/srep10657

2. Wallace DC. Mitochondria and cancer. Nat Rev Cancer (2012) 12:685-98. doi: $10.1038 / \mathrm{nrc} 3365$

3. Hamanaka RB, Chandel NS. Mitochondrial reactive oxygen species regulate hypoxic signaling. Curr Opin Cell Biol (2009) 21:894-9. doi:10.1016/j. ceb.2009.08.005

4. Semenza GL, Jiang BH, Leung SW, Passantino R, Concordet JP, Maire P, et al. Hypoxia response elements in the aldolase A, enolase 1, and lactate dehydrogenase A gene promoters contain essential binding sites for hypoxia-inducible factor 1. J Biol Chem (1996) 271:32529-37. doi:10.1074/ jbc.271.51.32529

5. Fukuda R, Zhang H, Kim JW, Shimoda L, Dang CV, Semenza GL. HIF-1 regulates cytochrome oxidase subunits to optimize efficiency of respiration in hypoxic cells. Cell (2007) 129:111-22. doi:10.1016/j.cell.2007.01.047

6. Kim JW, Tchernyshyov I, Semenza GL, Dang CV. HIF-1-mediated expression of pyruvate dehydrogenase kinase: a metabolic switch required for cellular adaptation to hypoxia. Cell Metab (2006) 3:177-85. doi:10.1016/j. cmet.2006.02.002

7. Chiche J, Rouleau M, Gounon P, Brahimi-Horn MC, Pouyssegur J, Mazure NM. Hypoxic enlarged mitochondria protect cancer cells from apoptotic stimuli. J Cell Physiol (2010) 222:648-57. doi:10.1002/jcp.21984

8. Al-Mehdi AB, Pastukh VM, Swiger BM, Reed DJ, Patel MR, Bardwell GC, et al. Perinuclear mitochondrial clustering creates an oxidant-rich nuclear biomarker for response to therapy (37). Further work exploring $\mathrm{CHCHD} 4$ 's role in regulating mitochondrial dynamics should reveal how $\mathrm{CHCHD} 4$ or specific $\mathrm{CHCHD} 4$ substrates control intracellular oxygenation, HIF signaling and the distribution of the mitochondrial network.

\section{ETHICS STATEMENT}

This study did not involve human participation, personal data, or use of human tissue.

\section{AUTHOR CONTRIBUTIONS}

LT designed and performed experiments, analyzed data, and contributed to writing the manuscript. OS generated stable CHCHD4 cell lines. MT performed electron microscopy analyses. MA provided the concept for the study, designed experiments, analyzed data, and wrote the manuscript.

\section{ACKNOWLEDGMENTS}

We thank all members of the Ashcroft laboratory, with particular thanks to Tom Briston for helpful discussions around determining mitochondrial endpoints.

\section{FUNDING}

The laboratory is funded in part by Cancer Research UK (CRUK), the Medical Research Council (MRC). LT was funded by MRC grants MR/K002201/1 and MR/K002201/2 and OS was funded by CR-UK grant C7358/A11223.

domain required for hypoxia-induced transcription. Sci Signal (2012) 5:ra47. doi:10.1126/scisignal.2002712

9. Yang J, Staples O, Thomas LW, Briston T, Robson M, Poon E, et al. Human $\mathrm{CHCHD} 4$ mitochondrial proteins regulate cellular oxygen consumption rate and metabolism and provide a critical role in hypoxia signaling and tumor progression. J Clin Invest (2012) 122:600-11. doi:10.1172/JCI58780

10. Fischer M, Riemer J. The mitochondrial disulfide relay system: roles in oxidative protein folding and beyond. Int J Cell Biol (2013) 2013:742923. doi:10.1155/2013/742923

11. Mesecke N, Terziyska N, Kozany C, Baumann F, Neupert W, Hell K, et al. A disulfide relay system in the intermembrane space of mitochondria that mediates protein import. Cell (2005) 121:1059-69. doi:10.1016/j.cell.2005. 04.011

12. Modjtahedi N, Tokatlidis K, Dessen P, Kroemer G. Mitochondrial proteins containing coiled-coil-helix-coiled-coil-helix $(\mathrm{CHCH})$ domains in health and disease. Trends Biochem Sci (2016) 41:245-60. doi:10.1016/j.tibs.2015. 12.004

13. Bihlmaier K, Mesecke N, Terziyska N, Bien M, Hell K, Herrmann JM. The disulfide relay system of mitochondria is connected to the respiratory chain. J Cell Biol (2007) 179:389-95. doi:10.1083/jcb.200707123

14. Hardie DG, Ross FA, Hawley SA. AMPK: a nutrient and energy sensor that maintains energy homeostasis. Nat Rev Mol Cell Biol (2012) 13:251-62. doi:10.1038/nrm3311

15. Kim H, Scimia MC, Wilkinson D, Trelles RD, Wood MR, Bowtell D, et al. Fine-tuning of Drp1/Fis1 availability by AKAP121/Siah2 regulates mitochondrial adaptation to hypoxia. Mol Cell (2011) 44:532-44. doi:10.1016/j. molcel.2011.08.045 
16. Li Y, Lim S, Hoffman D, Aspenstrom P, Federoff HJ, Rempe DA. HUMMR, a hypoxia- and HIF-1alpha-inducible protein, alters mitochondrial distribution and transport. J Cell Biol (2009) 185:1065-81. doi:10.1083/jcb. 200811033

17. Chau NM, Rogers P, Aherne W, Carroll V, Collins I, McDonald E, et al. Identification of novel small molecule inhibitors of hypoxia-inducible factor-1 that differentially block hypoxia-inducible factor-1 activity and hypoxiainducible factor-1alpha induction in response to hypoxic stress and growth factors. Cancer Res (2005) 65:4918-28. doi:10.1158/0008-5472.CAN-04-4453

18. Di Fonzo A, Ronchi D, Lodi T, Fassone E, Tigano M, Lamperti C, et al. The mitochondrial disulfide relay system protein GFER is mutated in autosomalrecessive myopathy with cataract and combined respiratory-chain deficiency. Am J Hum Genet (2009) 84:594-604. doi:10.1016/j.ajhg.2009.04.004

19. Becher D, Kricke J, Stein G, Lisowsky T. A mutant for the yeast scERV1 gene displays a new defect in mitochondrial morphology and distribution. Yeast (1999) 15:1171-81. doi:10.1002/(SICI)1097-0061(19990915) 15:12<1171::AID-YEA443>3.0.CO;2-T

20. Liu $X$, Hajnoczky G. Altered fusion dynamics underlie unique morphological changes in mitochondria during hypoxia-reoxygenation stress. Cell Death Differ (2011) 18:1561-72. doi:10.1038/cdd.2011.13

21. Smirnova E, Griparic L, Shurland DL, van der Bliek AM. Dynamin-related protein Drp1 is required for mitochondrial division in mammalian cells. $\mathrm{Mol}$ Biol Cell (2001) 12:2245-56. doi:10.1091/mbc.12.8.2245

22. Hagen T, Taylor CT, Lam F, Moncada S. Redistribution of intracellular oxygen in hypoxia by nitric oxide: effect on HIFlalpha. Science (2003) 302:1975-8. doi:10.1126/science. 1088805

23. Raleigh JA, Chou SC, Arteel GE, Horsman MR. Comparisons among pimonidazole binding, oxygen electrode measurements, and radiation response in C3H mouse tumors. Radiat Res (1999) 151:580-9. doi:10.2307/3580034

24. Arteel GE, Thurman RG, Raleigh JA. Reductive metabolism of the hypoxia marker pimonidazole is regulated by oxygen tension independent of the pyridine nucleotide redox state. Eur JBiochem (1998) 253:743-50. doi:10.1046/j.1432-1327.1998.2530743.x

25. Poon E, Harris AL, Ashcroft M. Targeting the hypoxia-inducible factor (HIF) pathway in cancer. Expert Rev Mol Med (2009) 11:e26. doi:10.1017/ S1462399409001173

26. Semenza GL. Hypoxia-inducible factors: mediators of cancer progression and targets for cancer therapy. Trends Pharmacol Sci (2012) 33:207-14. doi:10.1016/j.tips.2012.01.005

27. Kamentsky L, Jones TR, Fraser A, Bray MA, Logan DJ, Madden KL, et al. Improved structure, function and compatibility for CellProfiler: modular high-throughput image analysis software. Bioinformatics (2011) 27:1179-80. doi:10.1093/bioinformatics/btr095
28. Wai T, Langer T. Mitochondrial dynamics and metabolic regulation. Trends Endocrinol Metab (2016) 27:105-17. doi:10.1016/j.tem.2015.12.001

29. Pernas L, Scorrano L. Mito-morphosis: mitochondrial fusion, fission, and cristae remodeling as key mediators of cellular function. Annu Rev Physiol (2016) 78:505-31. doi:10.1146/annurev-physiol-021115-105011

30. Kuznetsov AV, Troppmair J, Sucher R, Hermann M, Saks V, Margreiter R. Mitochondrial subpopulations and heterogeneity revealed by confocal imaging: possible physiological role? Biochim Biophys Acta (2006) 1757:686-91. doi:10.1016/j.bbabio.2006.03.014

31. Ahmad T, Aggarwal K, Pattnaik B, Mukherjee S, Sethi T, Tiwari BK, et al. Computational classification of mitochondrial shapes reflects stress and redox state. Cell Death Dis (2013) 4:e461. doi:10.1038/cddis.2012.213

32. Rankin EB, Giaccia AJ. Hypoxic control of metastasis. Science (2016) 352:175-80. doi:10.1126/science.aaf4405

33. Nakazawa MS, Keith B, Simon MC. Oxygen availability and metabolic adaptations. Nat Rev Cancer (2016) 16:663-73. doi:10.1038/nrc.2016.84

34. Pekkurnaz G, Trinidad JC, Wang X, Kong D, Schwarz TL. Glucose regulates mitochondrial motility via Milton modification by O-GlcNAc transferase. Cell (2014) 158:54-68. doi:10.1016/j.cell.2014.06.007

35. Rambold AS, Kostelecky B, Elia N, Lippincott-Schwartz J. Tubular network formation protects mitochondria from autophagosomal degradation during nutrient starvation. Proc Natl Acad Sci U S A (2011) 108:10190-5. doi:10.1073/ pnas. 1107402108

36. Pouli D, Balu M, Alonzo CA, Liu Z, Quinn KP, Rius-Diaz F, et al. Imaging mitochondrial dynamics in human skin reveals depth-dependent hypoxia and malignant potential for diagnosis. Sci Transl Med (2016) 8:367ra169. doi:10.1126/scitranslmed.aag2202

37. Giedt RJ, Fumene Feruglio P, Pathania D, Yang KS, Kilcoyne A, Vinegoni C, et al. Computational imaging reveals mitochondrial morphology as a biomarker of cancer phenotype and drug response. Sci Rep (2016) 6:32985. doi: $10.1038 /$ srep32985

Conflict of Interest Statement: The authors declare that the research was conducted in the absence of any commercial or financial relationships that could be construed as a potential conflict of interest.

Copyright (c) 2017 Thomas, Staples, Turmaine and Ashcroft. This is an open-access article distributed under the terms of the Creative Commons Attribution License (CC BY). The use, distribution or reproduction in other forums is permitted, provided the original author(s) or licensor are credited and that the original publication in this journal is cited, in accordance with accepted academic practice. No use, distribution or reproduction is permitted which does not comply with these terms. 